\title{
Scan without evidence of dopaminergic deficit: A 10-year retrospective study
}

\author{
Nicolas Nicastro ${ }^{\text {a }}$, Valentina Garibotto ${ }^{\text {b, c }}$, Simon Badoud ${ }^{\text {a, d }}$, Pierre R. Burkhard ${ }^{\text {a, c, * }}$ \\ ${ }^{a}$ Department of Neurology, Geneva University Hospitals, Switzerland \\ ${ }^{\mathrm{b}}$ Department of Nuclear Medicine and Molecular Imaging, Geneva University Hospitals, Switzerland \\ c Faculty of Medicine, University of Geneva, Switzerland \\ d Physiology Unit, Department of Medicine, University of Fribourg, Switzerland
}

\begin{abstract}
Introduction: ${ }^{123}$ I-ioflupane SPECT is a powerful method to assess nigrostriatal dopamine system integrity. Several independent studies have shown that $1-15 \%$ of patients with suspected degenerative parkinsonism, mainly PD, have scans without evidence of dopaminergic deficit (SWEDD). It has been proposed that most SWEDD patients either present with a non-degenerative condition mimicking PD, such as atypical tremor or dystonia, or demonstrate an abnormal scan when repeated later. We here hypothesized that scan interpretation methods may also play a crucial yet underestimated role in this issue.

Methods: We previously established age-dependent reference values of striatal uptake by analyzing scans from a cohort of patients with non-degenerative conditions. We then studied a large population with well-established degenerative parkinsonism $(\mathrm{N}=410,80 \%$ with $\mathrm{PD})$, using identical imaging protocol, to evaluate the prevalence of patients with normal scans based on routine visual assessment. Each scan was eventually reassessed using the same automated method as for controls and a detailed 3D analysis.

Results: Ten potential SWEDD cases (2.4\%) were identified. However, both reassessment methods independently showed that these scans were all outside reference limits and/or visually abnormal when reexamined carefully, except for one case $(0.2 \%)$ with corticobasal syndrome.

Conclusion: SPECT misinterpretation emerges as an important contributor to the SWEDD population, suggesting that suspected SWEDD cases should prompt not only a serious diagnosis challenge but, equally important, a detailed scan reassessment. True SWEDD cases seem extremely rare in degenerative parkinsonism. We propose that the very concept of SWEDD is more confusing than helpful and should be definitely abandoned.
\end{abstract}

\section{Introduction}

${ }^{123}$ I-ioflupane (DaTSCAN ${ }^{\circledR}$ ) SPECT is one of the most commonly used molecular imaging methods to assess the integrity of the nigrostriatal dopamine system [1]. It has proven highly valuable to confirm degenerative parkinsonisms such as Parkinson's disease (PD), multiple system atrophy (MSA), progressive supranuclear palsy (PSP) and corticobasal degeneration (CBD) $[1,2,4]$.

In most studies, interpretation of ${ }^{123}$ I-ioflupane images has been

* Corresponding author. Department of Neurology, Geneva University Hospitals, Rue Gabrielle-Perret-Gentil 4, 1211 Geneva 14, Switzerland.

E-mail address: pierre.burkhard@hcuge.ch (P.R. Burkhard). based on visual assessment using a 0 (normal) to 3 scaling system $[2,3]$, the definition of each abnormal pattern being quite strictly defined. However, whereas this method has proved quite reliable $[4,5]$, its ordinal and relatively crude nature has left some room for intermediate or difficult-to-classify cases, in particular those with questionable uptake deficit early in the disease course. More recently, semi-quantitative methods of assessment have been developed based on the ratio of manually drawn or automatically delineated striatal regions or volumes of interest (ROIs, VOIs) to background activity [6,7] using various protocols and software. While less prone to subjectivity bias or to intra- and inter-observer variability, these analyses require robust reference values, usually established on healthy subjects, to which data from individual 
patients can be compared. However, generalizable reference values for semi-quantitative analyses are notoriously difficult to determine due to highly variable acquisition and reconstruction methods of SPECT imaging.

About a decade ago, several large drug trials on PD progression and neuroprotection reported that a significant proportion (between 1 and 15\%) of patients clinically diagnosed with PD showed a normal ${ }^{123}$ I-ioflupane SPECT uptake [8-11]. This unexpected finding was found consistent enough across studies to coin the acronym SWEDD for scan without evidence of dopaminergic deficit. Various hypotheses were proposed to explain the SWEDD phenomenon, including that SWEDD patients did not have PD or, alternatively and in complete contradiction with universally accepted neuropathological data, that very early PD may be associated with an unaltered nigrostriatal pathway or with highly effective compensatory mechanisms to counteract striatal dopamine decline. Over time, in the absence of clear explanations for it, the SWEDD concept has become popular and derived into a loose yet well accepted clinical entity, possibly a new neurological condition variably associating atypical tremor, dystonia, parkinsonism, gait disturbances, and various non-motor symptoms such as hyposmia. It soon became clear, however, that the SWEDD population was not uniform and included a heterogeneous group of patients, the vast majority of which did not fulfill PD clinical criteria but have conditions somehow resembling PD, such as essential tremor, dystonia, drug-induced or psychogenic parkinsonisms, vascular parkinsonism, fragile X-related tremor ataxia syndrome (FXTAS) or even aging-related extrapyramidal features. On the other hand, it appeared that a handful of SWEDD cases reported in the literature seem to have true PD. In those cases, the diagnosis of PD was eventually retained based on follow-up observations of disease progression, levodopa responsiveness or a repeated scan that was found abnormal [12-14]. Therefore, a normal scan in cases suspected to have a degenerative presynaptic parkinsonism, i.e. affecting the nigrostriatal system, may be seen in three distinct situations that could occur either alone or in combination: (1) clinical diagnosis is erroneous (i.e. patient has a non-degenerative condition); (2) scan interpretation is incorrect; and (3) indeed, degenerative presynaptic parkinsonism, notably PD, might be associated with a SWEDD pattern, at least early in the disease course, mechanisms of which remain to be established.

\section{Methods}

\subsection{Patients}

The present study has been conducted in compliance with the declaration of Helsinki. The protocol has been approved by our local ethics committee (CER 12-006R). As a retrospective observational study, no written consent was required. We collected data from $1005{ }^{123}$ I-ioflupane SPECT scans performed on 970 consecutive patients at our institution from October 2003 to September 2013. The vast majority of patients have been evaluated at least once by trained movement disorders specialists and the following data has been obtained: gender, age at disease onset, disease duration at the time of SPECT, body side predominance and final clinical diagnosis. Regarding degenerative parkinsonian syndromes, we used the UK Parkinson's Disease Study Brain Bank criteria for PD [15], the second consensus statement for the diagnosis of MSA [16], the National Institute of Neurological Disorders Society (NINDS) clinical research criteria for PSP [17] and the criteria for the diagnosis of CBD proposed by Armstrong et al. [18]. Patients were classified as having a non-degenerative parkinsonian syndrome or tremor, whenever they fulfilled the criteria for essential tremor [19], dystonic tremor [20], psychogenic parkinsonism [21] or drug-induced parkinsonism as described in Ref. [22]. Importantly, most patients were followed over several years and, in few instances, the diagnosis was changed according to the appearance of new features supporting another parkinsonian syndrome. In these cases, it is the last, more likely diagnosis which was retained. Finally, 33 patients had a second or even a third ${ }^{123}$ I-ioflupane SPECT scan and only the first one was considered for the collecting step of our study. Please refer to Fig. 1 for details of study enrollment.

\subsection{Imaging: acquisition and reconstruction}

${ }^{123}$ I-FP-CIT SPECT was performed according to the manufacturer's instructions. Patients received $185 \mathrm{MBq}$ of ioflupane in slow intravenous injection and were administered Lugol solution or sodium perchlorate for thyroid blockade. SPECT data acquisition started $4 \mathrm{~h}$ after ioflupane administration.

All scans were acquired on the same triple-head gamma camera (GCA-9300A/UI Toshiba Medical Systems AG, Oetwil am See, Switzerland) equipped with fan beam, low-energy, high-resolution collimators. Details of the acquisition and reconstruction are available elsewhere [22].

\subsection{Imaging: data analysis}

${ }^{123}$ I-ioflupane SPECT images were classified according to the standard 0-to-3 visual staging system [2,3], as available in the nuclear medicine report written at the time of scan.

\subsection{Determination of local semi-quantitative reference values}

Establishment of local reference values has been reported previously using a large cohort of patients with non-degenerative conditions and a normal ${ }^{123}$ I-ioflupane SPECT scan [22]. Importantly, it has been demonstrated that data obtained from this population were nearly identical with those from healthy subjects, thus validating the method. Regarding striatum, putamen $(\mathrm{P})$ and caudate nucleus (C) uptake values, cut-off points were taken at the $5^{\text {th }}$ percentile of the cohort of subjects with non-degenerative conditions. Uptake was considered as abnormal when below 3.93074-0.02156 $\times$ age for striatum, 3.79744-0.02168 $\times$ age for $P$ and 4.03099-0.02141 $\times$ age for C. For example, a 70-year-old patient is expected to have uptake values above 2.42 for striatum, 2.28 for $\mathrm{P}$ and 2.53 for $\mathrm{C}$. For striatal asymmetry index (AI) and C/P ratio, cut-off was established at the 95th percentile. Thus, a value above 12.22 was considered abnormal regarding striatal $\mathrm{AI}$, whereas $\mathrm{C} / \mathrm{P}$ ratio $>1.30$ indicated a pathological scan.

\subsection{Identification of SWEDD cases and scans reassessment}

We first collected all patients with a clinical diagnosis of degenerative parkinsonism and an initial SPECT scan considered normal and corresponding to a visual grade of 0 on the initial report (when the scan was performed). We then established automated BRASS $^{\text {TM }}$ semi-quantitative values for right and left C, P and striatum, as well as striatal $\mathrm{AI}$ and $\mathrm{C} / \mathrm{P}$ ratio and compared them to our local semi-quantitative age-dependent reference values. In parallel, two experts (one movement disorders specialist (PB) and one nuclear medicine specialist (VG), both well trained in ${ }^{123} \mathrm{I}$-ioflupane SPECT interpretation) reviewed in detail the visual staging of all scans in all planes using 3D images. Experts were blinded to the clinical diagnosis, as these grade-0 scans were reviewed among a collection of normal scans from patients with non-degenerative conditions independently prepared by another researcher (NN). At the end of this double cross-control assessment, we calculated the proportion of patients whose both visual staging review and 


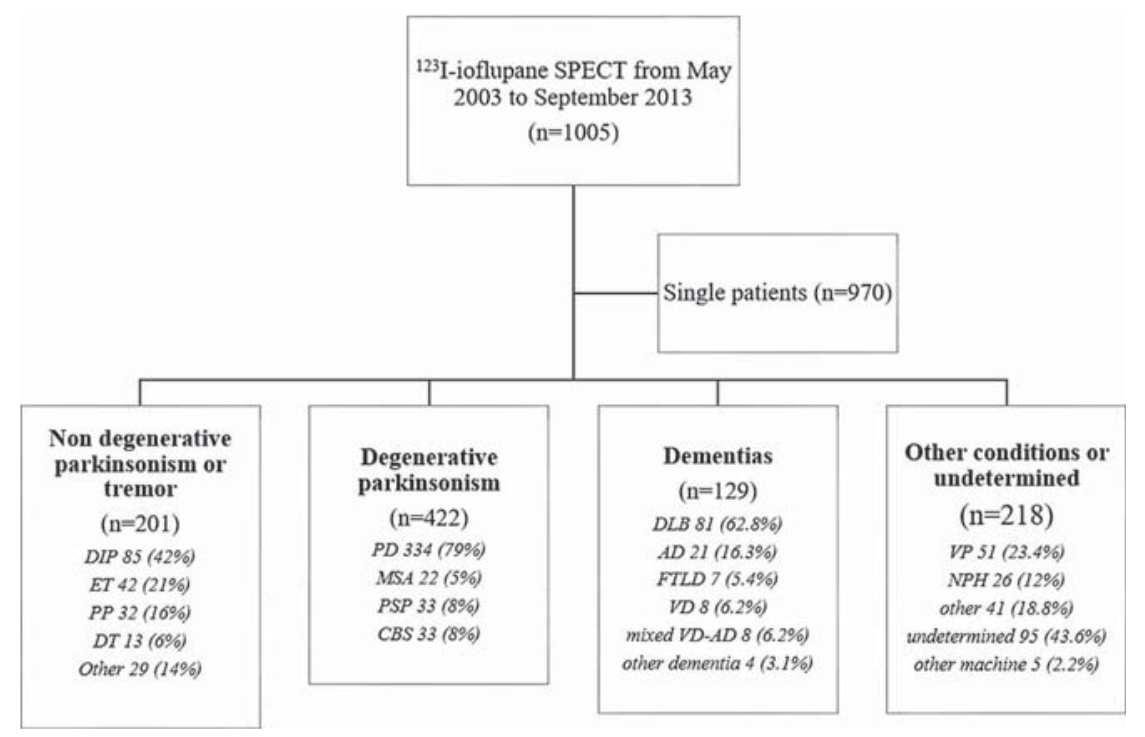

Fig. 1. Diagram showing the general categorization of patients who had a ${ }^{123}$ I-ioflupane SPECT in Geneva from 2003 to 2013 . DIP: drug-induced parkinsonism - ET: essential tremor - PP: psychogenic parkinsonism - DT: dystonic tremor - PD: Parkinson's disease - MSA: multiple-system atrophy - PSP: progressive supranuclear palsy - CBD: corticobasal degeneration - DLB: dementia with Lewy bodies - AD: Alzheimer's disease - FTLD: frontotemporal lobe dementia - VD: vascular dementia - VP: vascular parkinsonism - NPH: normal-pressure hydrocephalus.

-

semi-quantitative SPECT values were normal, representing definite SWEDD patients in our cohort. In addition, we applied our on-site reference values to the whole cohort to compare the results of the semi-quantitative method with the initial visual assessment. Finally, we assessed the evolution of images in those patients in whom more than one scan was performed.

-

\section{Results}

As shown in Fig. 1, 422 patients diagnosed with a degenerative parkinsonian syndrome were identified. Twelve subjects were excluded because tridimensional SPECT images were not available for re-analysis, so that BRASS ${ }^{\mathrm{TM}}$-derived semi-quantitative values could not be obtained. A total of 410 patients were eventually included in this study. Clinical diagnoses were PD $(n=322)$, MSA $(n=22)$, PSP $(n=33)$ and $\operatorname{CBD}(n=33)$. Mean age was $69.4 \pm 11.1$ years (range $25-92$ ), with a $51.6 \%$ male proportion, mean disease duration at the time of scan was $4.2 \pm 4.7$ years for PD, $2.9 \pm 2.2$ for MSA, $1.8 \pm 0.9$ for PSP and $2.5 \pm 1.5$ years for CBD patients. For PD patients, mean Hoehn \& Yahr $(\mathrm{H} \& \mathrm{Y})$ stage was estimated as $2.4 \pm 0.9$. Importantly, 136 subjects (33\%) from the whole cohort had a disease duration less than one year when scan was performed, including 103 (32\%) from the 322 PD patients, and 33 (37\%) from the 88 non-PD patients.

\subsection{Initial visual staging}

Ten patients (2.4\%) had an initial visual staging considered as normal (stage 0 ). Characteristics of these 10 patients, corresponding to typical SWEDD cases according to the literature, are summarized in Table 1 . Of note, a second ${ }^{123} \mathrm{I}$-ioflupane SPECT scan has been performed in 7 of these patients and for all 7, the repeated scan was clearly abnormal (stage 1 or 2 ).

Clinical diagnosis of patients with stage 0 SPECT was PD (50\%, 5 / $10)$, MSA (10\%, 1/10), PSP $(20 \%, 2 / 10)$ and CBD $(20 \%, 2 / 10)$, corresponding to a proportion to the whole diagnostic group of $1.6 \%$ for PD (5/322), 4.5\% for MSA (1/22), 6.1\% for PSP and for CBD (2/33 for each). Disease duration at the time of scan by diagnostic category was of $1.6 \pm 1.6$ years for PD (after excluding a single patient with a
10 -year evolution as an outlier), 0.5 year for the patient with MSA, $0.8 \pm 0.4$ for PSP and $1.5 \pm 0.7$ years for CBD. PD patients' mean H\&Y stage was $2.4 \pm 1.5$ (range $1-5$ ) at the time of scan. Disease duration at the time of scan for PD patients with normal initial visual assessment was significantly lower than that of the whole cohort of PD patients $(1.6 \pm 1.6$ years vs $4.2 \pm 4.7, \mathrm{p}<0.003$, Student $t$-test $)$. Regarding the cohort of provisional SWEDD, disease duration $(2.2 \pm 3.0$ years) was lower than that of the whole cohort of degenerative parkinsonism $(3.8 \pm 4.4, \mathrm{p}=0.12)$.

\subsection{Semi-quantitative BRASS ${ }^{\mathrm{TM}}$ analysis of SPECT with normal visual analysis}

Semi-quantitative evaluation of those 10 patients exhibiting a normal visual assessment showed that only 2 patients (20\%) had a strictly normal semi-quantitative assessment. For the 7 patients who had a second ${ }^{123}$ I-ioflupane SPECT scan, semi-quantitative analysis of the first scan already showed abnormal values for each of these cases $(7 / 7,100 \%)$. The two patients with initial normal visual assessment and unaltered semi-quantitative values carried a clinical diagnosis of CBD.

\subsection{Application of BRASS ${ }^{\mathrm{TM}}$-derived striatal uptake reference values for the whole cohort}

When applying our on-site reference values for C, P and striatum, including $\mathrm{AI}$ and $\mathrm{C} / \mathrm{P}$ ratio, on the whole cohort of patients with degenerative parkinsonism and available SPECT images $(n=410)$, only $10 / 410(2.4 \%)$ were considered to have a normal independent semi-quantitative assessment. Clinical characteristics, as well as initial and reassessed visual staging are summarized in Table 2. Regarding this group, disease duration was lower than that of the whole cohort $(2.1 \pm 2.1$ years vs $3.8 \pm 4.4, \mathrm{p}=0.11)$. Among these 10 patients, two had a concomitant stage 0 initial visual assessment, while eight patients had an abnormal (stage 1-2) visual staging. 
Table 1

Characteristics of patients with clinical degenerative parkinsonism and normal initial visual SPECT assessment, as well as their findings regarding semi-quantitative assessment and visual reassessment. A second ${ }^{123}$ I-ioflupane SPECT has been performed for patients whose number is followed by*.

\begin{tabular}{|c|c|c|c|c|c|c|c|}
\hline $\begin{array}{l}\text { Patient } \\
\text { number }\end{array}$ & Gender & Age (years) & Disease & $\begin{array}{l}\text { Disease duration at the time } \\
\text { of scan (years) }\end{array}$ & $\begin{array}{l}\text { Hoehn \& Yahr } \\
\text { scale }\end{array}$ & $\begin{array}{l}\text { Visual } \\
\text { reassessment } \\
\text { (stage) }\end{array}$ & $\begin{array}{l}\text { Semi-quantitative } \\
\text { evaluation }\end{array}$ \\
\hline $1^{*}$ & M & 78 & PD & 1 & 2 & 1 & Abnormal \\
\hline $2^{*}$ & M & 44 & Young-onset PD (PARK2) & 10 & 2 & 1 & Abnormal \\
\hline $3^{*}$ & M & 33 & Young-onset PD & 1 & 1 & 1 & Abnormal \\
\hline $4^{*}$ & $\mathrm{~F}$ & 68 & PD & 4 & 2 & 1 & Abnormal \\
\hline $5^{*}$ & $\mathrm{~F}$ & 68 & PD and DIP & 0.5 & 5 & 1 & Abnormal \\
\hline $6^{*}$ & M & 65 & MSA-P & 0.5 & & 1 & Abnormal \\
\hline $7^{*}$ & M & 70 & PSP & 0.5 & & 1 & Abnormal \\
\hline 8 & M & 69 & PSP & 1 & & 1 & Abnormal \\
\hline 9 & $\mathrm{~F}$ & 69 & CBD & 1 & & 1 & Normal \\
\hline \multirow[t]{2}{*}{10} & M & 56 & CBD & 2 & & 0 & Normal \\
\hline & & $\begin{array}{l}\text { Mean } 62.0 \pm 13.7 \\
\text { (range } 33-78 \text { ) }\end{array}$ & $\begin{array}{l}\text { 5/322 PD (1.6\%), } 1 / 22 \mathrm{MSA}(4.5 \%), 2 / 33 \text { PSP } \\
(6.1 \%), 2 / 33 \text { CBD }(6.1 \%)\end{array}$ & $\begin{array}{l}\text { Mean } 2.2 \pm 3.0 \text { (range } 0.5 \\
-10 \text { ) }\end{array}$ & $\begin{array}{l}\text { Mean } 2.4 \pm 1.5 \\
\text { (range } 1-5 \text { ) }\end{array}$ & & \\
\hline
\end{tabular}

Table 2

Characteristics of patients with clinical degenerative parkinsonism and normal semi-quantitative assessment. In addition, initial visual assessment, as well as visual review is mentioned.

\begin{tabular}{|c|c|c|c|c|c|c|}
\hline Number & Gender & Age & Disease & Disease duration (years) & Initial visual stage & Visual review \\
\hline 1 & $\mathrm{~F}$ & 88 & PD & 1 & 1 & \\
\hline 2 & $\mathrm{~F}$ & 79 & PD & 3 & 3 & \\
\hline 3 & $\mathrm{~F}$ & 65 & PD & 1 & 1 & \\
\hline 4 & $\mathrm{~F}$ & 89 & PD & 0.5 & 2 & \\
\hline 5 & $\mathrm{~F}$ & 79 & PD & 1 & 2 & \\
\hline 6 & $\mathrm{~F}$ & 68 & PD & 0.5 & 1 & \\
\hline 7 & M & 77 & PD & 7 & 1 & \\
\hline 8 & $\mathrm{~F}$ & 69 & CBD & 1 & 0 & 1 \\
\hline 9 & $\mathrm{~F}$ & 76 & CBD & 4 & 2 & \\
\hline \multirow[t]{2}{*}{10} & M & 56 & CBD & 2 & 0 & 0 \\
\hline & $\mathrm{M} / \mathrm{F}$ ratio $2 / 8=0.25$ & & $7 / 10$ PD 3/10 CBD & $2.5 \pm 2.2($ range $1-7)$ & $1.3 \pm 0.9($ range $0-3)$ & \\
\hline
\end{tabular}

\subsection{Review of visual staging}

In parallel, two experts in ${ }^{123}$ I-ioflupane SPECT analysis reassessed the visual stage of the 10 patients with a potential SWEDD status, based on the initial visual stage. Only $1 / 10$ (10\%) exhibited a definitely normal (stage 0 ) visual assessment. This patient was among the two with normal semi-quantitative values. Potential causes of stage misattribution that were identified by the experts included: misalignment of scan images, heterogeneity of uptake patterns and underestimation of $P$ size.

\subsection{Definite SWEDD patients}

Taking together both evaluations methods (semi-quantitative analysis and expert review of scans), only one patient (1/410, $0.24 \%)$ with a degenerative form of parkinsonism had a strictly normal ${ }^{123}$ I-ioflupane SPECT. At the time of scan, this 56-year-old male patient exhibited a rapidly progressive, highly asymmetrical nontremulous akinetic-rigid syndrome associated with parietal lobetype sensory symptoms including ideo-motor apraxia and alien limb phenomenon, which were all unresponsive to high doses of levodopa and dopamine agonists, thus fulfilling a diagnosis of probable CBD. Axial images of his SPECT scan are shown in Fig. 2. Of note, none of the 322 PD patients could be labeled as SWEDD, even those 103 cases where scans were performed very early, less than a year after disease onset.

\section{Discussion}

From one of the largest ${ }^{123}$ I-ioflupane SPECT-assessed cohort of degenerative parkinsonism patients studied yet, we identified 10 cases (2.4\% of 410 patients) in whom routine visual interpretation yielded a normal scan result, corresponding to typical SWEDD cases as reported in the literature. An extensive, two-step reassessment of these scans, using the automated semi-quantitative BRASS ${ }^{\mathrm{TM}}$ software and locally established reference values as well as diagnosis-blinded detailed expert reviews, showed that all but one SWEDD cases ( $0.24 \%$ of 410 patients) with CBD actually had a definitely abnormal ${ }^{123}$ I-ioflupane SPECT and not a single patient with PD could be considered a true SWEDD, casting serious doubts about published estimates of the real prevalence of SWEDD in degenerative parkinsonism and, in general, about the very usefulness of the SWEDD concept.

Mean age of the 10 patients with a provisional SWEDD label was similar to that of our global cohort of degenerative parkinsonisms, yet they had a trend toward a shorter disease duration at the time of scan $(2.2 \pm 3.0$ vs $3.8 \pm 4.4$ years, $\mathrm{p}=0.12)$.

Scans from this population of 10 provisional SWEDD cases were then extensively reassessed. First, applying semi-quantitative assessment yielded only two subjects with normal values. This supports our early hypothesis that routine visual assessment alone may not be reliable enough to define scan normality, at least in some cases with borderline uptakes, as already shown in a previous study [22]. Second, a detailed review of the visual stage by two experts showed that only one of these 10 patients could be considered as having a definite normal (stage 0 ) visual assessment. This further comforts the notion that visual inspection is prone to inter-observer variability and that the level of expertise in assessing scans may be crucial in the final staging attribution. Third, in seven cases, a second ${ }^{123}$ I-ioflupane SPECT scan was repeated a few years later because of disease progression that was felt inconsistent with the initial scan graded as normal. For all these seven patients, not 


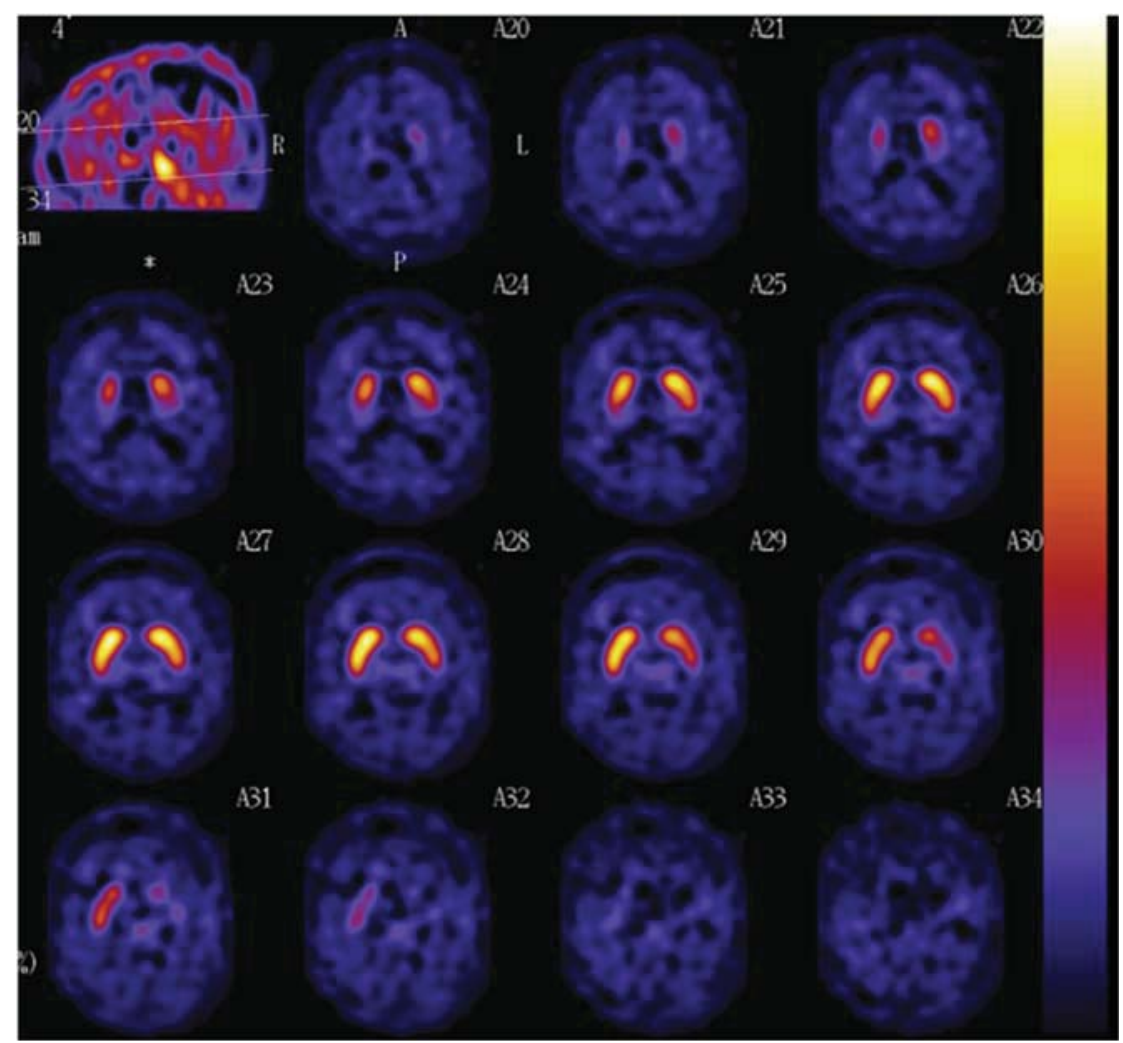

Fig. 2. Axial ${ }^{123}$ I-ioflupane SPECT images of the only patient in our cohort with clinical degenerative parkinsonism (probable corticobasal degeneration) and normal SPECT.

only was the second scan considered as definitely abnormal (stage 1 or 2), thus confirming the clinical suspicion of a degenerative condition, but, interestingly, uptake values of some VOIs of the first scan were also below reference limits. In these cases, a correct interpretation of the first scan would likely have spared the second one. Sensitivity of initial visual and semi-quantitative analyses is similar when considered independently. However, assessments taken in combination allow a greater sensitivity for determination of nigro-striatal impairment.

In previous studies, an incorrect initial diagnosis of degenerative parkinsonism seems to account for a significant proportion of SWEDDs $[12,13,23]$ and, in those studies where a second, follow-up scan has been performed, conversion from normal to abnormal was rather uncommon (8.3\% in Ref. [13] and $12.5 \%$ in Ref. [24]). Conversely, at the 22-month follow-up time point of the PRECEPT study [13], 3 out of 629 cases with an initial abnormal scan $(0.5 \%)$ switched to a normal category, implying that the first scan was incorrectly assigned an abnormal label. However, in none of the studies mentioned above was the quality of the scan interpretation challenged or even discussed, yet the need for semi-quantitative values has been strongly suggested in recent publications $[4,6]$, further supported by the finding of a direct correlation between dopamine transporter uptake and neuromelanin-positive nigral cell count on pathological specimens [25]. Technical issues (misalignment of scan, medication interfering with dopaminergic uptake of SPECT) can also play a role. With respect to the diagnostic issue, it is important to underline that virtually all patients included in our study were followed over time, usually for many years, and this allowed disease progression, the main criterion supporting a degenerative process at the basis of the observed clinical features, to be captured. In addition, the vast majority of patients were managed by movement disorders specialists and clinical diagnoses were established according to the more recent, validated and widely used criteria. For example, in the case of PD, the presence of a tremor alone, even at rest, did not qualify for a diagnosis of PD and such patients were not even enrolled in this study.

In this series, SWEDD were more common in CBD patients (6.1\%) compared to the other forms of degenerative parkinsonism. This may not be unexpected as it is well known that CBD neurodegeneration tends to start in cortical areas whereas basal ganglia are implicated later in the disease [26], possibly accounting for higher DAT uptake early in the disease course. This is not totally surprising nor is it new, as at least two cases of pathology-proven CBD with normal dopaminergic SPECT have been published so far [27,28].

In conclusion, our study strongly suggests that a high proportion of SWEDD cases may be related to an incorrect visual interpretation of ${ }^{123}$ I-ioflupane SPECT scans, rather than or in addition to an erroneous clinical diagnosis. Interestingly, we were unable to identify a single case of PD associated with a normal scan thanks to our combined reassessment, even very early in the disease course, fully supporting the recently proposed MDS clinical criteria for PD, where a normal scan is considered an absolute exclusion criterion [29]. Based on our results and the confusing literature surrounding it, we concur with other researchers [30] that the very concept of SWEDD is clinically and scientifically highly problematic and should be definitely abandoned.

\section{Acknowledgments}

None.

\section{References}

[1] J. Booij, J.D. Speelman, M.W. Horstink, E.C. Wolters, The clinical benefit of imaging striatal dopamine transporters with [123I]FP-CIT SPET in 
differentiating patients with presynaptic parkinsonism from those with other forms of parkinsonism, Eur. J. Nucl. Med. 28 (2001) 266-272.

[2] T.S. Benamer, J. Patterson, D.G. Grosset, J. Booij, K. de Bruin, E. van Royen, J.D. Speelman, M.H. Horstink, H.J. Sips, R.A. Dierckx, J. Versijpt, D. Decoo, C. Van Der Linden, D.M. Hadley, M. Doder, A.J. Lees, D.C. Costa, S. Gacinovic W.H. Oertel, O. Pogarell, H. Hoeffken, K. Joseph, K. Tatsch, J. Schwarz, V. Ries, Accurate differentiation of parkinsonism and essential tremor using visual assessment of [123I]-FP-CIT SPECT imaging: the [123I]-FP-CIT study group, Mov. Disord. 15 (2000) 503-510.

[3] A.M. Catafau, E. Tolosa, D.C.U.P.S.S. Group, Impact of dopamine transporter SPECT using 123I-Ioflupane on diagnosis and management of patients with clinically uncertain Parkinsonian syndromes, Mov. Disord. 19 (2004) 1175-1182.

[4] N. Papathanasiou, P. Rondogianni, P. Chroni, M. Themistocleous, E. Boviatsis X. Pedeli, D. Sakas, I. Datseris, Interobserver variability, and visual and quantitative parameters of (123)I-FP-CIT SPECT (DaTSCAN) studies, Ann. Nucl. Med. 26 (3) (2012) 234-240.

[5] S. Ottaviani, M. Tinazzi, I. Pasquin, W. Nothdurfter, G. Tomelleri, E. Fincati, G. Nordera, G. Moretto, A. Fiaschi, N. Smania, P. Giorgetti, A. Antonini, Comparative analysis of visual and semi-quantitative assessment of striatal [123I]FP-CIT-SPET binding in Parkinson's disease, Neurol. Sci. 27 (2006) 397-401.

[6] A. Skanjeti, T. Angusti, M. Margheron, M. Iudicello, V. Podio, FP-CIT SPECT evaluation: time to go beyond visual assessment!, Eur. J. Nucl. Med. Mol. Imaging 39 (2012) 727-728.

[7] A. Davidsson, C. Georgiopoulos, N. Dizdar, G. Granerus, H. Zachrisson, Comparison between visual assessment of dopaminergic degeneration pattern and semi-quantitative ratio calculations in patients with Parkinson's disease and Atypical Parkinsonian syndromes using DaTSCAN SPECT, Ann. Nucl. Med. 28 (9) (2014) 851-859.

[8] Parkinson Study Group, A randomized controlled trial comparing pramipexole with levodopa in early Parkinson's disease: design and methods of the CALMPD Study, Clin. Neuropharmacol. 23 (1) (2000) 34-44.

[9] S. Fahn, D. Oakes, I. Shoulson, K. Kieburtz, A. Rudolph, A. Lang, C.W. Olanow, C. Tanner, K. Marek, G. Parkinson Study, Levodopa and the progression of Parkinson's disease, N. Engl. J. Med. 351 (24) (2004) 2498-2508.

[10] A.L. Whone, R.L. Watts, A.J. Stoessl, M. Davis, S. Reske, C. Nahmias, A.E. Lang, O. Rascol, M.J. Ribeiro, P. Remy, W.H. Poewe, R.A. Hauser, D.J. Brooks, Slower progression of Parkinson's disease with ropinirole versus levodopa: the REALPET study, Ann. Neurol. 54 (1) (2003) 93-101.

[11] PRECEPT Investigators Parkinson Study Group, Mixed lineage kinase inhibitor CEP-1347 fails to delay disability in early Parkinson disease, Neurology 69 (15) (2007) 1480-1490.

[12] M.A. Utiumi, A.C. Felicio, C.R. Borges, V.L. Braatz, S.A. Rezende, R.P. Munhoz, R.A. Bressan, H.B. Ferraz, H.A. Teive, Dopamine transporter imaging in clinically unclear cases of parkinsonism and the importance of scans without evidence of dopaminergic deficit (SWEDDs), Arq. Neuropsiquiatr. 70 (9) (2012) 667-673.

[13] K. Marek, J. Seibyl, S. Eberly, D. Oakes, I. Shoulson, A.E. Lang C. Hyson, D. Jennings, Longitudinal follow-up of SWEDD subjects in the PRECEPT Study, Neurology 82 (20) (2014) 1791-1797.

[14] M. Menendez-Gonzalez, F. Tavares, N. Zeidan, J.M. Salas-Pacheco, O. AriasCarrion, Diagnoses behind patients with hard-to-classify tremor and normal DaT-SPECT: a clinical follow up study, Front. Aging Neurosci. 6 (2014) 56.

[15] A.J. Hughes, S.E. Daniel, L. Kilford, A.J. Lees, Accuracy of clinical diagnosis of idiopathic Parkinson's disease: a clinico-pathological study of 100 cases, J. Neurol. Neurosurg. Psychiatry 55 (3) (1992) 181-184.
[16] S. Gilman, G.K. Wenning, P.A. Low, D.J. Brooks, C.J. Mathias, J.Q. Trojanowski, N.W. Wood, C. Colosimo, A. Durr, CJ. Fowler, H. Kaufmann, T. Klockgether, A. Lees, W. Poewe, N. Quinn, T. Revesz, D. Robertson, P. Sandroni, K. Seppi, M. Vidailhet, Second consensus statement on the diagnosis of multiple system atrophy, Neurology 71 (9) (2008) 670-676.

[17] I. Litvan, Y. Agid, D. Calne, G. Campbell, B. Dubois, R.C. Duvoisin, C.G. Goetz, L.I. Golbe, J. Grafman, J.H. Growdon, M. Hallett, J. Jankovic, N.P. Quinn, E. Tolosa, D.S. Zee, Clinical research criteria for the diagnosis of progressive supranuclear palsy (Steele-Richardson-Olszewski syndrome): report of the NINDS-SPSP international workshop, Neurology 47 (1) (1996) 1-9.

[18] M.J. Armstrong I. Litvan, A.E. Lang T.H. Bak, K.P. Bhatia, B. Borroni, A.L. Boxer, D.W. Dickson, M. Grossman, M. Hallett, K.A. Josephs, A. Kertesz, S.E. Lee, B.L. Miller, S.G. Reich, D.E. Riley, E. Tolosa, A.I. Troster, M. Vidailhet, W.J. Weiner, Criteria for the diagnosis of corticobasal degeneration, Neurology 80 (5) (2013) 496-503.

[19] G. Deuschl, P. Bain, M. Brin, Consensus statement of the movement disorde society on tremor. Ad hoc scientific committee, Mov. Disord. 13 (Suppl. 3) (1998) 2-23.

[20] A. Albanese, F. Asmus, K.P. Bhatia, A.E. Elia, B. Elibol, G. Filippini, T. Gasser, J.K. Krauss, N. Nardocci, A. Newton, J. Valls-Sole, EFNS guidelines on diagnosis and treatment of primary dystonias, Eur. J. Neurol. 18 (1) (2011) 5-18.

[21] F. Morgante, M.J. Edwards, A.J. Espay, A. Fasano, P. Mir, D. Martino, Diagnostic agreement in patients with psychogenic movement disorders, Mov. Disord. 27 (4) (2012) 548-552.

[22] N. Nicastro, V. Garibotto, A. Poncet, S. Badoud, P.R. Burkhard, Establishing onsite reference values for ${ }^{123}$ I-FP-CIT SPECT (DaTSCAN(R)) using a cohort of individuals with non-degenerative conditions, Mol. Imaging Biol. 18 (2) (2016) 302-312.

[23] F. Sixel-Doring, K. Liepe, B. Mollenhauer, E. Trautmann, C. Trenkwalder, The role of ${ }^{123}$ I-FP-CIT-SPECT in the differential diagnosis of Parkinson and tremor syndromes: a critical assessment of 125 cases, J. Neurol. 258 (12) (2011) 2147-2154.

[24] A. Batla, R. Erro, M. Stamelou, S.A. Schneider, P. Schwingenschuh, C. Ganos, K.P. Bhatia, Patients with scans without evidence of dopaminergic deficit: a long-term follow-up study, Mov. Disord. 29 (14) (2014) 1820-1825.

[25] J. Kraemmer, G.G. Kovacs, L. Perju-Dumbrava, S. Pirker, T. Traub-Weidinger W. Pirker, Correlation of striatal dopamine transporter imaging with post mortem substantia nigra cell counts, Mov. Disord. 29 (14) (2014) 1767-1773.

[26] B.F. Boeve D.M. Maraganore J.E. Parisi, J.E. Ahlskog N. Graff-Radford, R.J. Caselli, D.W. Dickson, E. Kokmen, R.C. Petersen, Pathologic heterogeneity in clinically diagnosed corticobasal degeneration, Neurology 53 (4) (1999) $795-800$.

[27] V. Kaasinen, M. Gardberg, M. Roytta, M. Seppanen, M. Paivarinta, Normal dopamine transporter SPECT in neuropathologically confirmed corticobasal degeneration, J. Neurol. 260 (5) (2013) 1410-1411.

[28] S.S. O'Sullivan, D.J. Burn, J.L. Holton, A.J. Lees, Normal dopamine transporter single photon-emission CT scan in corticobasal degeneration, Mov. Disord. 23 (16) (2008) 2424-2426.

[29] R.B. Postuma, D. Berg M. Stern, W. Poewe C.W. Olanow W. Oertel, J. Obeso, K. Marek, I. Litvan, A.E. Lang, G. Halliday, C.G. Goetz, T. Gasser, B. Dubois, P. Chan, B.R. Bloem, C.H. Adler, G. Deuschl, MDS clinical diagnostic criteria for Parkinson's disease, Mov. Disord. 30 (12) (2015) 1591-1601.

[30] R. Erro, S.A. Schneider, M. Stamelou, N.P. Quinn, K.P. Bhatia, What do patients with scans without evidence of dopaminergic deficit (SWEDD) have? New evidence and continuing controversies, J. Neurol. Neurosurg. Psychiatry 87 (3) (2016) 319-323. 